\title{
ON INNER PRODUCT SPACES OVER DEDEKIND DOMAINS OF CHARACTERISTIC TWO
}

\author{
RICHARD C. WAGNER
}

\begin{abstract}
Suppose $D$ is a Dedekind domain of characteristic 2 and $(M, \varphi)$ is an inner product space, i.e. $M$ is a finitely generated projective $D$ module supplied with a nonsingular symmetric bilinear form $\varphi$. It is shown that $(M, \varphi)$ is determined up to isometry by the extension of $\varphi$ to $F \otimes_{D} M$, where $F$ is the quotient field of $D$, and the value module $2(M)$ of all $\varphi(m, m)$ for $m$ in $M$. In particular, a hyperbolic space $\mathbf{H}(M)$ is completely determined by the rank of the finitely generated projective module $M$. As consequences, genera coincide with isometry classes, and if $N_{1}$ and $N_{2}$ are isometric nonsingular submodules of $(M, \varphi)$ such that $\mathscr{2}\left(N_{1}^{\perp}\right)=\mathscr{2}\left(N_{2}^{\perp}\right)$, then $N_{1}^{\perp}$ and $N_{2}^{\perp}$ are isometric. Also, given an $F$ inner product space $(V, \varphi)$ and a $D^{(2)}$ submodule $P$ of $D$, a necessary and sufficient condition is given for the existence of a $D$ inner product space $(M, \Psi)$ such that $(F M, \Psi) \cong(V, \varphi)$ and $\mathscr{Q}(M)=P$.
\end{abstract}

1. Preliminaries. Unless otherwise stated, $D$ is a Dedekind domain of characteristic 2 with quotient field $F$ and all $D$ modules will be left $D$ modules. $\mathfrak{A}, \mathfrak{B}, \mathfrak{E}, \ldots$ are fractional ideals of $D$, and $\mathfrak{A}^{-1}=\{x \in F \mid x \mathfrak{A} \subseteq D\}$ is the inverse of the fractional ideal $\mathfrak{A}$.

If $M$ is a finitely generated projective $D$ module, an inner product on $M$ is a nonsingular symmetric bilinear form $\varphi$ on $M$. In this case $(M, \varphi)$ will be called an inner product space. If $F M$ denotes the $F$ vector space $F \otimes_{D} M$, then $\varphi$ has a unique extension to a nonsingular symmetric bilinear form on $F M$. We also use $\varphi$ to denote this extension, so $(F M, \varphi)$ is also an inner product space.

We use $D^{(2)}\left(F^{(2)}\right)$ to denote the subring of $D(F)$ of all squares. If $(M, \varphi)$ and $(F M, \varphi)$ are inner product spaces, then $\mathscr{Q}(M)=\{\varphi(m, m) \mid m \in M\}$ and $\mathscr{Q}(F M)$ $=\{\varphi(x, x) \mid x \in F M\}$ are subgroups of $D$ and $F$, respectively. They become $D^{(2)}$ or $F^{(2)}$ submodules when we define $a^{2} \cdot \varphi(m, m)=\varphi(a m, a m)$ for $a$ in $D$ or $F$, or they become $D$ or $F$ submodules when we define $a \circ \varphi(m, m)=\varphi(a m, a m) . \mathscr{2}(M)$ and $\mathscr{2}(F M)$ are called the value modules of the respective inner product spaces. We note that $q_{\rho}: M \rightarrow \mathscr{Q}(M)$, defined by $q_{\varphi}(m)=\varphi(m, m)$, is a $D$ module homomorphism when $\mathcal{Q}(M)$ is viewed as a $D$ module.

If $P$ is a projective module over an arbitrary commutative ring and $k$ is an arbitrary symmetric bilinear form on $P$, we let $P^{*}=\operatorname{Hom}_{D}(P, D)$ and define the inner product space $\mathbf{M}(P)=\left(P \oplus P^{*}, h_{P}\right)$ by $h_{P}(x+f, y+g)=k(x, y)+g(x)$ $+f(y), x, y \in P, f, g \in P^{*}$. Any inner product space isometric to a $\mathbf{M}(P)$ is called

Received by the editors March 23, 1982.

1980 Mathematics Subject Classification. Primary 10C02, 10C03, 15A63; Secondary 13B30, 13C10, $13 \mathrm{~F} 30$.

Key words and phrases. Symmetric bilinear forms, value modules, hyperbolic and metabolic spaces, Witt cancellation, genera, Dedekind and discrete valuation rings. 
a metabolic (or split) space. It is easy to see that an inner product space $(M, \varphi)$ is metabolic if and only if there is a submodule $N$ of $M$ which is a direct summand of $M$ and $N$ is precisely equal to its orthogonal complement $N^{\perp}$. If the symmetric bilinear form $k$ is identically zero, then we denote $\mathbf{M}(P)$ by $\mathbf{H}(P)$. Any inner product space isometric to a $\mathbf{H}(P)$ is said to be a hyperbolic space. We note that (0) $\oplus P^{*}$ is totally isotropic in both $\mathbf{M}(P)$ and $\mathbf{H}(P)$, while $P \oplus(0)$ is also totally isotropic in $\mathbf{H}(P)$. If $P$ is a free $D$ module on one generator then $\mathbf{H}(P)$ is called a hyperbolic plane, and if $x, y \in \mathbf{H}(P)$ are such that $h_{P}(x, x)=0=h_{P}(y, y)$ and $h_{P}(x, y)=1$, then $x, y$ is called a hyperbolic basis for the plane.

Proofs for the following over an arbitrary commutative ring can be found in [4].

1.1. If $P$ is a projective $D$ module and $P=P_{1} \oplus P_{2}$, then $\mathbf{M}(P) \cong \mathbf{M}\left(P_{1}\right) \perp \mathbf{M}\left(P_{2}\right)$. In particular, $\mathbf{H}(P) \cong \mathbf{H}\left(P_{1}\right) \perp \mathbf{H}\left(P_{2}\right)$.

1.2. If $(P, \varphi)$ is an inner product space then $(P, \varphi) \perp(P,-\varphi) \cong \mathbf{M}(P)$.

We now introduce a mapping similar to that of A. Roy [9]; also see [12]. Suppose $(X, \varphi)$ is an inner product space where $X=\mathbf{M}(P) \perp Q$. If $\alpha: P \rightarrow Q$ is a $D$ module homomorphism we define

$$
E_{\alpha}: \mathbf{M}(P) \perp Q \rightarrow \mathbf{M}(P) \perp Q
$$

by

$$
\begin{array}{ll}
E_{\alpha}(x)=x+\alpha(x), & x \in P, \\
E_{\alpha}(f)=f, & f \in P^{*}, \\
E_{\alpha}(y)=y-\alpha^{*}(y), & y \in Q .
\end{array}
$$

Here $\alpha^{*}: Q \rightarrow P^{*}$ is defined by

$$
\alpha^{*}={ }^{t} a \cdot d_{\varphi},
$$

where $d_{\varphi}: Q \rightarrow Q^{*},{ }^{t} \alpha: Q^{*} \rightarrow P^{*}$ are defined by $d_{\varphi}(z)=\varphi(, z)$ and ${ }^{t} \alpha$ is the dual of $\alpha$.

The following is easily established.

1.3(i) $E_{\alpha}$ restricted to $Q$ is an isometry onto $E_{\alpha}(Q)$.

(ii) $\mathbf{M}(P) \perp Q=E_{\alpha}(\mathbf{M}(P)) \perp E_{\alpha}(Q)$.

1.4 THEOREM. Given $\mathbf{M}(P)$ and $(Q, \varphi)$ inner product spaces with $\mathscr{Q}(\mathbf{M}(P)) \subseteq \mathscr{Q}(Q)$, we have

$$
\mathbf{M}(P) \perp(Q, \varphi) \cong \mathbf{H}(P) \perp(Q, \varphi) .
$$

Proof. We have a $D$ module homomorphism $h: Q \rightarrow \mathscr{Q}(Q)$ defined by $h(x)=$ $\varphi(x, x)$. Because $\mathscr{2}(Q)$ is a torsion free, finitely generated $D$ module, it is a projective $D$ module. Consequently, $Q=Q_{1} \oplus K$, where $K$ is the kernel of $h$. Let $h^{-1}: \mathscr{Q}(Q) \rightarrow Q_{1}$ denote the inverse of $h$ restricted to $Q_{1}$. If $k_{P}$ is the symmetric bilinear form on $P$ in $\mathbf{M}(P)$ we let $k: P \rightarrow \mathscr{2}(\mathbf{M}(P))$ be the $D$ module homomorphism defined by $k(x)=k_{P}(x, x)$, and let $\alpha: P \rightarrow Q$ be defined by $\alpha=h^{-1} k$. We note that if $x \in P$ then $k(x)=h(\alpha(x))$. Therefore $E_{\alpha}(x)=x+\alpha(x)$ is isotropic and, consequently, $E_{\alpha}(P)$ is totally isotropic. By 1.3,

$$
\mathbf{M}(P) \perp Q=E_{\alpha}(\mathbf{M}(P)) \perp E_{\alpha}(Q),
$$

and $E_{\alpha}(\mathbf{M}(P))$ is isometric to $\mathbf{H}(P)$ and $E_{\alpha}(Q)$ is isometric to $Q$. 
2. Cancellation and classification. The following argument, due to A. Frohlich [2], shows that Witt cancellation over Dedekind domains of characteristic zero is not always possible.

Let $\mathfrak{A}$ and $\mathfrak{B}$ be fractional ideals of the Dedekind domain $D$. Because

$$
D \oplus \mathfrak{A} \cong \mathfrak{B}^{-1} \oplus \mathfrak{B} \mathfrak{A} \text { and } \mathfrak{B} \oplus \mathfrak{B} \mathfrak{A} \cong D \oplus \mathfrak{B}^{2} \mathfrak{A},
$$

we have

$$
\begin{aligned}
\mathbf{H}(D) \perp \mathbf{H}(\mathfrak{A}) & \cong \mathbf{H}(D \oplus \mathfrak{U}) \cong \mathbf{H}\left(\mathfrak{B}^{-1} \oplus \mathfrak{B} \mathfrak{A}\right) \cong \mathbf{H}\left(\mathfrak{B}^{-1}\right) \perp \mathbf{H}(\mathfrak{B} \mathfrak{U}) \\
& \cong \mathbf{H}(\mathfrak{B}) \perp \mathbf{H}(\mathfrak{B} \mathfrak{A}) \cong \mathbf{H}(\mathfrak{B} \oplus \mathfrak{B} \mathfrak{A}) \cong \mathbf{H}\left(D \oplus \mathfrak{B}^{2} \mathfrak{U}\right) \\
& \cong \mathbf{H}(D) \perp \mathbf{H}\left(\mathfrak{B}^{2} \mathfrak{A}\right) .
\end{aligned}
$$

If the characteristic of $D$ is zero then Frohlich shows that $\mathbf{H}(\mathfrak{A})$ is not always isometric to $\mathbf{H}\left(\mathfrak{B}^{2} \mathfrak{U}\right)$. Thus $\mathbf{H}(D)$ cannot be cancelled from both sides.

The situation for Dedekind domains of characteristic two is quite different, as the following illustrates.

2.1 TheOREM. Let $D$ be a Dedekind domain of characteristic two and $M$ a finitely generated, projective $D$ module. If $n$ is the rank of $M$ then $\mathbf{H}(M) \cong \mathbf{H}\left(D^{n}\right)$.

Proof. Because $M$ can be expressed as a direct sum of fractional ideals of $D$, it suffices to show that $\mathbf{H}(\mathfrak{A}) \cong \mathbf{H}(D)$ when $\mathfrak{A}$ is a fractional ideal of $D$. Because $\mathfrak{U}^{-1}=\operatorname{Hom}_{D}(\mathfrak{A}, D), \mathbf{H}(\mathfrak{A})$ has $\mathfrak{A} \oplus \mathfrak{A}^{-1}$ for its module and $h_{\mathfrak{A}}: \mathfrak{A} \times \mathfrak{A}^{-1} \rightarrow D$ for its symmetric bilinear form. By [1, Theorem 22.11], $\mathfrak{A} \oplus \mathfrak{A}^{-1}$ is isomorphic to a free $D$ module of rank two, so there are $x$ and $y$ in $\mathfrak{A} \oplus \mathfrak{U}^{-1}$ so that $\mathfrak{U} \oplus \mathfrak{U}^{-1}=D x \oplus D y$. Because $\mathscr{Q}(\mathbf{H}(\mathfrak{A}))=(0), x$ and $y$ must both be isotropic, and because $\mathbf{H}(\mathfrak{U})$ is nonsingular, $h_{\mathfrak{A}}(x, y)$ must be a unit of $D$. Therefore $\mathbf{H}(\mathfrak{U}) \cong \mathbf{H}(D)$.

The preceding result raises the possibility that cancellation of hyperbolic direct summands is possible over Dedekind domains of characteristic two. In order to prove this, we need the following lemmas.

2.2 LemMA. Let $(X, \varphi)$ be an inner product space over $D$ and $X=H \perp M$, where $H$ is a hyperbolic plane with hyperbolic basis $x, y$. If $z \in M$ and $H^{\prime}=D x \oplus D(y+z)$ then there is a submodule $M^{\prime}$ of $X$ isometric to $M$ such that $X=H^{\prime} \perp M^{\prime}$.

2.3 LEMMA. Let $(X, \varphi)$ be an inner product space over $D$ such that $H_{1} \perp M=X=$ $H_{2} \perp N$, where $H_{1}$ and $H_{2}$ are hyperbolic planes. Suppose $H_{1}$ and $H_{2}$ have hyperbolic bases $x, y$ and $w, z$, respectively. If $\varphi(x, w)=u$, an invertible element of $D$, then $M$ and $N$ are isometric.

Proofs can be found in [11]. Also see [7, pp. 256-257 and 3, Theorem 40].

The following establishes cancellation of hyperbolic spaces.

2.4 TheOREM. Suppose $(X, \varphi)$ is an inner product space over $D$ such that $H_{1} \perp M=$ $X=H_{2} \perp N$, where $H_{1}$ and $H_{2}$ are isometric hyperbolic spaces. Then $M$ and $N$ are isometric. 
Proof. Because of Theorem 2.1 it suffices to prove the theorem when $H_{1}$ and $H_{2}$ are hyperbolic planes. Let $x, y$ and $w, z$ be hyperbolic bases for $H_{1}$ and $H_{2}$, respectively. If $\varphi\left(H_{1}, H_{2}\right)=(0)$ then $H_{1} \subseteq N$ and there is a submodule $N^{\prime}$ of $N$ such that $N=H_{1} \perp N^{\prime}$. Now

$$
H_{1} \perp M=X=H_{1} \perp\left(H_{2} \perp N^{\prime}\right),
$$

so by Lemma 2.3, $M$ and $H_{2} \perp N^{\prime}$ are isometric. But $H_{2} \perp N^{\prime}$ and $H_{1} \perp N^{\prime}$ are isometric, so $M$ and $N$ are isometric.

Thus we can assume $\varphi\left(H_{1}, H_{2}\right) \neq(0)$ and, without loss of generality, $\varphi\left(w, H_{1}\right) \neq$ (0). We wish to reduce to the case in which $\varphi\left(w, H_{1}\right)=D$. Suppose $\varphi\left(w, H_{1}\right)=\mathfrak{A} \neq$ $D$. We have $z=h+m$, where $h \in H_{1}, m \in M$ and $m$ must be isotropic. But $\varphi(w, z)=1$, so $\mathfrak{A}+\varphi(w, m) D=D$. Let $\varphi(w, x)=a, \varphi(w, y)=b$ and $\varphi(w, m)=$ $c$. Then $D a+D b+D c=D$. Because $\mathfrak{A} \neq(0)$, either $a \neq 0$ or $b \neq 0$; say $a \neq 0$. Let $\bar{D}=D / D a$ and let $\bar{x}$ represent the image of $x \in D$ in $\bar{D}$. But $\bar{D}$ is a finite direct sum of fields and local principal ideal rings [14, p. 278] and, consequently, has stable range one, i.e. because $\bar{D} \bar{b}+\bar{D} \bar{c}=\bar{D}$ there is $\bar{r} \in \bar{D}$ such that $\bar{D}(\bar{b}+\bar{r} \bar{c})=\bar{D}$. Therefore $D a+D(b+r c)=D$. Now $H_{3}=D x \oplus D(y+r m)$ is a hyperbolic plane and by Lemma 2.2 there is a submodule $M^{\prime}$ of $X$, isometric to $M$, such that $X=H_{3} \perp M^{\prime}$ and $\varphi\left(w, H_{3}\right)=D$. Thus we can assume from the beginning that $\varphi\left(w, H_{1}\right)=D$. Now define a $D$ module homomorphism $f: D x \oplus D y \rightarrow D$ by $f(u)=$ $\varphi(w, u)$, so $f$ is onto $D$. Suppose $x^{\prime} \in D x \oplus D y$ with $f\left(x^{\prime}\right)=1$. Then $x^{\prime}$ is isotropic and $D x \oplus D y=D x^{\prime} \oplus K$, where $K$ is the kernel of $f$. Now $K$ must be free of rank 1, say $K=D y^{\prime}$. But $y^{\prime}$ is isotropic, so by multiplying $y^{\prime}$ by an invertible element of $D$, if necessary, $x^{\prime}, y^{\prime}$ is a hyperbolic basis for $H_{1}=D x \oplus D y$. Because $\varphi\left(w, x^{\prime}\right)=1$, we have $M$ and $N$ isometric by Lemma 2.3.

We are now in a position to characterize inner product spaces by their extensions to the quotient field $F$ of $D$ and their value modules. The analogous result for local rings of characteristic two has been established by C. H. Sah [10, p. 268]. Also see [7, p. 259].

2.5 TheORem. Let $(M, \varphi)$ and $(N, \Psi)$ be inner product spaces such that $(F M, \varphi)$ and $(F N, \Psi)$ are isometric. Then $(M, \varphi)$ and $(N, \Psi)$ are isometric if and only if $\mathscr{2}(M)=\mathscr{2}(N)$.

Proof. If $(M, \varphi)$ and $(N, \Psi)$ are isometric it is clear that $\mathscr{2}(M)=\mathscr{2}(N)$. For the converse, we assume that $(F M, \varphi)$ and $(F N, \Psi)$ are isometric and $\mathscr{Q}(M)=\mathscr{2}(N)$.

Let $X=M \perp N \perp N$ be provided with the direct sum of the corresponding inner products. By Theorems 1.4 and 2.1,

$$
X \cong M \perp \mathbf{M}(N) \cong M \perp \mathbf{H}(N) \cong M \perp \mathbf{H}\left(D^{n}\right),
$$

where $n$ is the rank of $N$.

On the other hand, $F M \perp F N$ has a totally isotropic subspace $V$ of dimension $n$ such that $V=V^{\perp}$. Let $M_{0}=V \cap(M \perp N)$, so $M_{0}$ is a pure submodule of $M \perp N$ and, consequently, a totally isotropic direct summand of $M \perp N$ of rank $n$. Suppose $M \perp N=M_{0} \oplus M_{1}$, so $M_{1}$ has rank $n$. Then $M_{1}$ must be anisotropic, for otherwise $F M \perp F N$ would have a totally isotropic subspace of dimension greater than $n$. 
Because $V=V^{\perp}$, we have $M_{0}=M_{0}^{\perp}$ and, consequently, $M \perp N \cong \mathbf{M}\left(M_{1}\right)$. If $M_{1} \cong D^{n-1} \oplus \mathfrak{A}$, where $\mathfrak{A}$ is an integral ideal, then

$$
\begin{aligned}
\mathbf{H}\left(\mathfrak{U}^{-1}\right) \perp X & =\mathbf{H}\left(\mathfrak{A}^{-1}\right) \perp M \perp N \perp N \\
& \cong \mathbf{H}\left(\mathfrak{A}^{-1}\right) \perp \mathbf{M}\left(D^{n-1}\right) \perp \mathbf{M}(\mathfrak{A}) \perp N \cong \mathbf{M}\left(D^{n+1}\right) \perp N,
\end{aligned}
$$

because $\mathbf{H}\left(\mathfrak{U}^{-1}\right) \perp \mathbf{M}(\mathfrak{A}) \cong \mathbf{M}\left(D^{2}\right)$. But $\mathscr{Q}\left(\mathbf{M}\left(D^{n+1}\right)\right)=\mathscr{Q}(M)=\mathscr{2}(N)$, so by Theorem 1.4,

$$
\mathbf{M}\left(D^{n+1}\right) \perp N \cong \mathbf{H}\left(D^{n+1}\right) \perp N
$$

Thus

$$
\mathbf{H}\left(D^{n+1}\right) \perp N \cong \mathbf{H}\left(\mathfrak{U}^{-1}\right) \perp X \cong \mathbf{H}\left(D^{n+1}\right) \perp M .
$$

By Theorem 2.4, $M$ and $N$ are isometric.

In particular, a $D$ inner product space $(M, \varphi)$ is hyperbolic if and only if $\mathscr{Q}(M)=(0)$.

If $\mathfrak{B}$ is a maximal ideal of $D$ then the completion of $F$ with respect to the $\mathfrak{B}$-adic valuation will be denoted by $\hat{F}_{\mathfrak{B}}$ and the valuation ring by $\hat{D}_{\mathfrak{B}}$. If $(M, \varphi)$ is a $D$ inner product space, we let $\hat{M}_{\mathfrak{B}}=\hat{D}_{\mathfrak{B}} \otimes_{D} M$ and let $\varphi_{\mathfrak{B}}$ be the extension of $\varphi$ to $\hat{M}_{\mathfrak{B}}$.

2.6 Corollary. If $(M, \varphi)$ and $(N, \Psi)$ are two inner product spaces over $D$, $(F M, \varphi)$ is isometric to $(F N, \Psi)$ and $\left(\hat{M}_{\mathfrak{B}}, \varphi_{\mathfrak{B}}\right)$ is isometric to $\left(\hat{N}_{\mathfrak{B}}, \Psi_{\mathfrak{R}}\right)$ for all maximal ideals $\mathfrak{B}$ of $D$, then $(M, \varphi)$ and $(N, \Psi)$ are isometric.

Proof. If $\mathfrak{B}$ is a maximal ideal of the Dedekind domain $D$ then $D^{(2)}$ is also a Dedekind domain with quotient field $F^{(2)}$ and maximal ideal $\mathfrak{B}^{(2)}$. We denote the completion of $F^{(2)}$ with respect to th: $\mathfrak{B}^{(2)}$-adic valuation by $\hat{F}_{\mathfrak{B}}^{(2)}$ and the valuation ring by $\hat{D}_{\mathfrak{B}}^{(2)}$. It is easy to see that $\hat{F}_{\mathfrak{B}}^{(2)}$ is the subfield of $\hat{F}_{\mathfrak{B}}$ of squares and $\hat{D}_{\mathfrak{P}}^{(2)}$ is the subring of $\hat{D}_{\mathfrak{B}}$ of squares.

If $\mathscr{Q}(M)$ is viewed as a $D^{(2)}$ module, by [8, Theorem 5.3] we have

$$
\mathscr{Q}(M)=\bigcap\left[\mathscr{Q}(M) \otimes F^{(2)} \cap \mathscr{Q}(M) \otimes \hat{D}_{\mathfrak{B}}^{(2)}\right],
$$

where the tensor products are over $D^{(2)}$ and the intersection ranges over all maximal ideals $\mathfrak{B}$ of $D$. The corollary now follows from the theorem and the observation that

$$
\begin{aligned}
& \mathscr{Q}(M) \otimes_{D^{(2)}} F^{(2)}=\mathscr{2}\left(M \otimes_{D} F\right)=\mathscr{Q}(F M), \\
& \mathscr{Q}(M) \otimes_{D^{(2)}} \hat{D}_{\mathfrak{P}}^{(2)}=\mathscr{2}\left(M \otimes_{D} \hat{D}_{\mathfrak{P}}\right)=\mathscr{2}\left(\hat{M}_{\mathfrak{B}}\right) .
\end{aligned}
$$

2.7 CoROllaRY. Let $(X, \varphi)$ be a $D$ inner product space and $M$ and $N$ nonsingular isometric submodules so that $M \perp M^{\perp}=X=N \perp N^{\perp}$. If $\mathscr{Q}\left(M^{\perp}\right)=\mathscr{2}\left(N^{\perp}\right)$ then $M^{\perp}$ and $N^{\perp}$ are isometric.

Proof. We have

$$
F M \perp F M^{\perp}=F X=F N \perp F N^{\perp} .
$$

Because $\mathscr{Q}\left(M^{\perp}\right)=\mathscr{2}\left(N^{\perp}\right)$, we also have $\mathscr{2}\left(F M^{\perp}\right)=\mathscr{Q}\left(F N^{\perp}\right)$. By [12, Theorem 2.3], $F M^{\perp}$ and $F N^{\perp}$ are isometric and, consequently, $M^{\perp}$ and $N^{\perp}$ are isometric.

In [12, Theorem 2.3] it was shown that when $D$ is a field of characteristic $2, M^{\perp}$ being anisotropic is sufficient to guarantee cancellation. We give an example to show that this is not always the case for discrete valuation rings of characteristic 2 . 
Let $R=K[[\pi]]$ be the ring of formal power series in the indeterminate $\pi$ over the field $K=Z_{2}(t)$ of rational functions in the indeterminate $t$ over the field $Z_{2}$ of two elements. We claim that

where $u=1+\pi$.

$$
\langle 1\rangle \perp\left[\begin{array}{c}
\pi^{2}, 1 \\
1, t
\end{array}\right] \cong\langle 1\rangle \perp\left[\begin{array}{c}
\pi^{2}, u \\
u, t+1
\end{array}\right],
$$

If, on the left-hand side, $x$ is a basis for the one-dimensional direct summand and $y, z$ is a basis for the two-dimensional direct summand, then on the right-hand side, $x+w$ is a basis for the one-dimensional summand and $y+\pi w, z+x+w$, where $w=y+\pi x$, is a basis for the two-dimensional summand. It is easy to see that the two-dimensional summands are anisotropic and they cannot be isometric because they have different value models. Thus the one-dimensional summand $\langle 1\rangle$ cannot be cancelled.

The existence question remains; namely, given an $F$ inner product space $(V, \varphi)$ and a $D^{(2)}$ submodule $P$ of $D$, is there a $D$ inner product space $(M, \Psi)$ such that $(F M, \Psi) \cong(V, \varphi)$ and $\mathscr{Q}(M)=P$ ?

To answer this question, we introduce some terminology. If $(V, \varphi)$ is an $F$ inner product space, we say that a $D$ lattice $M$ in $V$ is integral if $\varphi$ restricted to $M$ takes values only in $D$, and we say that it is nonsingular if, in addition, $\varphi$ restricted to $M$ is nonsingular.

Given $(M, \varphi)$, an anisotropic $D$ inner product space with $Q=\mathscr{Q}(M)$, a $D^{(2)}$ submodule of $D$, we define

$$
B_{\varphi}: Q \times Q \rightarrow D^{(2)} \quad \text { by } \quad B_{\varphi}(a, b)=\varphi(x, y)^{2} \text {, }
$$

where $a=\varphi(x, x), b=\varphi(y, y)$ for some $x, y \in M$. It is easy to see that $B_{\varphi}$ is a well-defined symmetric bilinear form with $B_{\varphi}(a, a)=a^{2}$ when $a \in \mathscr{Q}(M)$. Furthermore:

2.8 LEMMA. (i) ( $\left.\mathscr{Q}(M), B_{\varphi}\right)$ is a $D^{(2)}$ inner product space.

(ii) Two anisotropic $D$ inner product spaces $(M, \varphi)$ and $(N, \Psi)$ are isometric if and only if $\mathscr{Q}(M)=\mathscr{Q}(N)$ and $B_{\varphi}=B_{\Psi}$.

(iii) If $L$ is a $D$ lattice in $(F M, \varphi)$, we associate to it the $D^{(2)}$ lattice $2(L)$ of $\left(\mathscr{Q}(F M), B_{\varphi}\right)$. This establishes a one-to-one correspondence between integral $D$ lattices in $(F M, \varphi)$ and integral $D^{(2)}$ lattices in $\left(\mathscr{2}(F M), B_{\varphi}\right)$ and between corresponding nonsingular lattices.

Proof. If $a \in D^{(2)}$, we use $\sqrt{a}$ to denote the unique element $b$ of $D$ such that $b^{2}=a$. If $q_{\varphi}: M \rightarrow \mathscr{Q}(M)$ is the $D$ module homomorphism defined by $q_{\varphi}(m)=$ $\varphi(m, m)$, then $B_{\varphi}\left(q_{\varphi}(m), q_{\varphi}(n)\right)=\varphi(m, n)^{2}$ for $m, n \in M$.

To prove (i) assume $f \in \operatorname{Hom}_{D^{(2)}}\left(2(M), D^{(2)}\right)$. Then $f^{*}(m)=\sqrt{f(\varphi(m, m))}$ is in $\operatorname{Hom}_{D}(M, D)$ so there is $x \in M$ such that $f^{*}=\varphi(x$,$) and, consequently, f=$ $B_{\varphi}\left(q_{\varphi}(x)\right.$, ).

In order to prove (ii) assume first that $(M, \varphi)$ and $(N, \Psi)$ are isometric. Then we clearly have $\mathscr{Q}(M)=\mathscr{2}(N)$ and, if $f: M \rightarrow N$ is an isometry, $\varphi(m, n)=$ $\Psi(f(m), f(n))$ for $m, n \in M$. Consequently,

$$
B_{\varphi}\left(q_{\varphi}(m), q_{\varphi}(n)\right)=B_{\Psi}\left(q_{\Psi}(f(m)), q_{\Psi}(f(n))\right),
$$


so $B_{\varphi}=B_{\Psi}$. Conversely, assume $\mathscr{2}(M)=\mathscr{2}(N)$ and $B_{\varphi}=B_{\Psi}$. Because $(N, \Psi)$ is anisotropic, $q_{\Psi}$ is invertible and $q_{\Psi}^{-1} q_{\varphi}$ is easily seen to be the required isometry.

It is easy to verify the first two assertions of (iii). To prove the third, assume $B_{\varphi}$ is nonsingular on $2(M)$ and we are given $f \in \operatorname{Hom}_{D^{(2)}}(L, D)$. Then $f^{*}=\left(f q_{\varphi}^{-1}\right)^{2} \in$ $\operatorname{Hom}_{D^{(2)}}\left(\mathscr{Q}(L), D^{(2)}\right)$ and there is $a \in \mathscr{Q}(L)$ such that $f^{*}=B_{\varphi}(a$,$) . But then$ $f=\varphi\left(q_{\varphi}^{-1}(a), \quad\right)$ and, consequently, $\varphi$ is nonsingular on $L$. Conversely, if we assume $\varphi$ is nonsingular on $L$ and we are given $f^{*} \in \operatorname{Hom}_{D^{(2)}}\left(\mathscr{Q}(L), D^{(2)}\right)$, then $f=\sqrt{f^{*} q_{\varphi}}$ $\in \operatorname{Hom}_{D}(L, D)$ and there is $x \in L$ such that $f=\varphi(x$,$) . Then f^{*}=B_{\varphi}\left(q_{\varphi}(x), \quad\right)$ so $B_{\varphi}$ is nonsingular on $\mathscr{Q}(L)$.

Given an anisotropic $D$ inner product space $(M, \varphi)$, we call $\left(\mathscr{Q}(M), B_{\varphi}\right)$ the Wall invariant of $(M, \varphi)$. See [13, Lemma 3.4.2]. Also, recall from [12, p. 375] that $V=H \perp V_{1} \perp V_{1} \perp V_{0}$ is said to be a Witt decomposition of the $F$ inner product space $(V, \varphi)$ if $H$ is a hyperbolic space, $V_{1}$ and $V_{0}$ are anisotropic and $\mathscr{Q}\left(V_{1}\right) \cap \mathscr{Q}\left(V_{0}\right)$ $=(0)$. Also see $[4$, p. 33 and 5, p. 66].

We are now in a position to answer the existence question.

2.9 TheOREM. Given an $F$ inner product space $(V, \varphi)$ with Witt decomposition $V=H \perp V_{1} \perp V_{1} \perp V_{0}$ and $a D^{(2)}$ submodule $P$ of $D$, there is a $D$ inner product space $(M, \Psi)$ such that $\mathscr{Q}(M)=P$ and $(F M, \Psi) \cong(V, \varphi)$ if and only if

(i) $F^{(2)} P=\mathscr{Q}(V)$,

(ii) there is a maximal nonsingular $D^{(2)}$ lattice $L$ in the Wall invariant $\left(\mathscr{Q}\left(V_{0}\right), B_{\varphi}\right)$ of $\left(V_{0}, \varphi\right)$ which is contained in $P$ such that $\left(P \cap \mathscr{Q}\left(V_{0}\right)\right) / L$ is a torsion module and is $a$ direct sum of no more than $\frac{1}{2} \operatorname{dim} H$ cyclic $D^{(2)}$ modules.

Moreover, the ideal class of $M$ is equal to the ideal class of $L$ when it is viewed as a $D$ module.

Proof. We first assume that there is a $D$ inner product space $(M, \Psi)$ with $(F M, \Psi) \cong(V, \varphi)$ and $\mathscr{Q}(M)=P$. Then we clearly have $F^{(2)} P=\mathscr{Q}(V)$. Because $D$ is a Dedekind domain, $M=\mathbf{M}\left(M_{1}\right) \perp M_{0}$, where $M_{0}$ is anisotropic. Then

$$
F \mathbf{M}\left(M_{1}\right) \perp F M_{0} \cong V=H \perp V_{1} \perp V_{1} \perp V_{0},
$$

so $F M_{0} \cong V_{0}$ by [12, Theorem 2.5]. Consequently, $2\left(F M_{0}\right)=\mathscr{2}\left(V_{0}\right)$ and $B_{\Psi}=B_{\varphi}$ by Lemma 2.8. Therefore $\left(\mathscr{Q}\left(M_{0}\right), B_{\Psi}\right)$ is a nonsingular $D^{(2)}$ lattice on $\left(\mathscr{Q}\left(V_{0}\right), B_{\varphi}\right)$ which is contained in $P$. Suppose $L$ is a nonsingular lattice in $\left(\mathscr{Q}\left(V_{0}\right), B_{\varphi}\right)$ containing $\mathscr{2}\left(M_{0}\right)$ and contained in $P$. Then $\left(L, B_{\varphi}\right)$ is a $D^{(2)}$ inner product space so there is a submodule $L_{0}$ of $L$ such that

$$
L=L_{0} \perp \mathscr{Q}\left(M_{0}\right), \quad \text { so } \quad F^{(2)} L=F^{(2)} L_{0} \perp F^{(2)} \mathscr{Q}\left(M_{0}\right) .
$$

But $F^{(2)} \mathscr{Q}\left(M_{0}\right)=\mathscr{Q}\left(F M_{0}\right)=\mathscr{2}\left(V_{0}\right)$ so $F^{(2)} L_{0}=(0)$ and $L=\mathscr{Q}\left(M_{0}\right)$. Therefore $\mathscr{2}\left(M_{0}\right)$ is a maximal nonsingular lattice in the Wall invariant $\left(\mathscr{Q}\left(V_{0}\right), B_{\varphi}\right)$ of $\left(V_{0}, \varphi\right)$.

By Theorem 1.4, we can assume that $M_{1}$ has no direct summand $N$ such that $\mathscr{2}(N) \neq(0)$ and $\mathscr{Q}(N) \subseteq \mathscr{Q}\left(M_{0}\right)$. Let

$$
N_{2}=\left\{m \in M_{1} \mid \Psi(m, m) \in D \cap \mathscr{Q}\left(V_{0}\right)\right\} .
$$


Then $N_{2}$ is a pure submodule of $M_{1}$ so there is a submodule $N_{1}$ of $M_{1}$ so that $M_{1}=N_{1} \oplus N_{2}$. By Theorem 1.1, $\mathbf{M}\left(M_{1}\right) \cong \mathbf{M}\left(N_{1}\right) \perp \mathbf{M}\left(N_{2}\right)$. Also, $P \cap \mathscr{Q}\left(V_{0}\right)=$ $\mathscr{2}\left(N_{2}\right)+\mathscr{Q}\left(M_{0}\right)$ and $\mathscr{2}\left(N_{2}\right) \subseteq F^{(2)} \mathscr{Q}\left(M_{0}\right)$. By the Invariant Factor Theorem [1, p. 150], there are elements $m_{1}, \ldots, m_{n}$ in $\mathscr{Q}\left(M_{0}\right)$ such that

$$
\mathscr{Q}\left(M_{0}\right)=\mathfrak{\Im}_{1} m_{1} \oplus \cdots \oplus \mathfrak{\Im}_{n} m_{n} \text { and } \mathscr{Q}\left(N_{2}\right)=\mho_{1} \mathfrak{\Im}_{1} m_{1} \oplus \cdots \oplus \mho_{n} \mathfrak{\Im}_{n} m_{n},
$$

where the $\mathfrak{\Im}_{i}$ and $\mho_{i}$ are fractional ideals of $D^{(2)}$ and $\xi_{1} \supseteq \varpi_{2} \supseteq \cdots \supseteq \xi_{n}$ are called the invariant factors. If $k$ is the rank of $\mathscr{2}\left(N_{2}\right)$ as a $D$ module, $\mathbb{E}_{i}=(0)$ for $i>k$. Because of our observation regarding direct summands of $M_{1}$, $F_{i}$ is not an integral ideal for $i \leqslant k$. Therefore $\mathfrak{\Im}_{i} m_{i} \subseteq \mathbb{F}_{i} \mathfrak{\Im}_{i} m_{i}$ for $i \leqslant k$ and

$$
\left(P \cap \mathscr{Q}\left(V_{0}\right)\right) / \mathscr{Q}\left(M_{0}\right) \cong \mathfrak{F}_{1} \mathfrak{\Im}_{1} / \mathfrak{\Im}_{1} \oplus \cdots \oplus \mathfrak{F}_{k} \mathfrak{\Im}_{k} / \mathfrak{\Im}_{k} \text {. }
$$

By the Invariant Factor Theorem and Theorem 4.12 of $\left[8\right.$, p. 48], each $\mathfrak{F}_{i} \widetilde{\Im}_{i} / \widetilde{\Im}_{i}$ is cyclic. Also, $F \mathbf{M}\left(N_{2}\right) \perp F M_{0}$ is isometric to a subspace of $H \perp V_{0}$ and $\operatorname{dim} F M_{0}=$ $\operatorname{dim} V_{0}$ so $2 k \leqslant \operatorname{rank} \mathbf{M}\left(N_{2}\right) \leqslant \operatorname{dim} H$.

To prove the converse, we assume (i) and (ii) are satisfied. We first consider the case in which $H=(0)$ and $V_{0}=(0)$, so $V=V_{1} \perp V_{1}$. We proceed by induction on the rank of $P$ as a $D^{(2)}$ module. If the rank of $P$ is one, we can identify $P$ with a fractional ideal of $D^{(2)}$ which we will view as a $D$ module using $r \circ a=r^{2} a$ for $r \in D$ and $a \in P$. Define $h: P \times P \rightarrow D$ by $h(a, b)=\sqrt{a b}$. Then $h$ is a symmetric bilinear form and $\mathscr{Q}(\mathbf{M}(P))=P$. Because $\mathscr{Q}\left(F^{(2)} \mathbf{M}(P)\right)=F^{(2)} P=\mathscr{2}\left(V_{1}\right), F^{(2)} \mathbf{M}(P) \cong V_{1} \perp$ $V_{1}$ by Theorem 2.5 (iii) [12, p. 382]. In the case that $P$ has rank greater than one, we can represent $P$ as a direct sum of two nonzero submodules, use the induction hypothesis on each, and piece together the results to prove the assertion for $P$.

We now consider the general case. By Lemma 2.8(iii) there is a nonsingular $D$ lattice $M_{0}$ in $V_{0}$ such that $\mathscr{Q}\left(M_{0}\right)=L$, so $\left(F M_{0}, \varphi\right)=\left(V_{0}, \varphi\right)$. Now $P \cap \mathscr{Q}\left(V_{0}\right)$ is a pure submodule of $P$ so $P=\left(P \cap \mathscr{Q}\left(V_{0}\right)\right) \oplus Q$ for some $D^{(2)}$ submodule $Q$ of $P$. Let $\left(\mathbf{M}\left(U_{1}\right), \Psi_{1}\right)$ be an $F$ inner product space with $\mathscr{Q}\left(U_{1}\right)=F^{(2)} Q$ and having no hyperbolic part in its Witt decomposition. By the initial argument there is a $D$ inner product space $\left(\mathbf{M}\left(M_{1}\right), \Psi_{2}\right)$ such that $\left(F \mathbf{M}\left(M_{1}\right), \Psi_{2}\right) \cong\left(\mathbf{M}\left(U_{1}\right), \Psi_{1}\right)$ and $2\left(\mathbf{M}\left(M_{1}\right)\right)$ $=Q$. Because $\left(P \cap \mathscr{Q}\left(V_{0}\right)\right) / L$ is a torsion module, $F^{(2)} L=F^{(2)}\left(P \cap \mathscr{Q}\left(V_{0}\right)\right)$ and, consequently, $F^{(2)} L \oplus F^{(2)} Q=\mathscr{Q}(V)$. By Theorem 2.5 of [12, p. 382] we have

$$
F \mathbf{M}\left(M_{1}\right) \perp F M_{0} \cong V_{1} \perp V_{1} \perp V_{0} .
$$

Now let $S$ be a maximal submodule of $L$ which is a direct summand of $P \cap \mathscr{Q}\left(V_{0}\right)$, so $P \cap \mathscr{Q}\left(V_{0}\right)=T \oplus S$ for some submodule $T$ of $P \cap \mathscr{2}\left(V_{0}\right)$. Because $S \subseteq L \subseteq P \cap$ $\mathscr{Q}\left(V_{0}\right)$ we also have $L=(L \cap T) \oplus S$ and $\left(P \cap \mathscr{Q}\left(V_{0}\right)\right) / L \cong T /(L \cap T)$. If $T$ and $L \cap T$ had a common direct summand it would be a direct summand of $P \cap \mathscr{Q}\left(V_{0}\right)$, which would be contained in $L$, contradicting the maximality of $S$. By the Invariant Factor Theorem and (ii), $\operatorname{rank}(L \cap T)=\operatorname{rank} T \leqslant \frac{1}{2} \operatorname{dim} H$. Now let $\left(\mathbf{M}\left(U_{2}\right), \Psi_{3}\right)$ be an $F$ inner product space which has no hyperbolic part in its Witt decomposition and $\mathscr{Q}\left(U_{2}\right)=F^{(2)} T$. By the initial argument there is a $D$ inner product space $\left(\mathbf{M}\left(M_{2}\right), \Psi_{4}\right)$ such that $\left(F \mathbf{M}\left(M_{2}\right), \Psi_{4}\right) \cong\left(\mathbf{M}\left(U_{2}\right), \Psi_{3}\right)$ and $\mathscr{Q}\left(\mathbf{M}\left(M_{2}\right)\right)=T$. Because $F^{(2)} T \subseteq \mathscr{Q}\left(V_{0}\right)$,

$$
F \mathbf{M}\left(M_{2}\right) \perp F M_{0} \cong F \mathbf{H}\left(M_{2}\right) \perp F M_{0}
$$


by Theorem 1.4. Now let $n=\frac{1}{2} \operatorname{dim} H$-rank $T$,

$$
M=\mathbf{H}\left(D^{n}\right) \perp \mathbf{M}\left(M_{2}\right) \perp \mathbf{M}\left(M_{1}\right) \perp M_{0},
$$

and let $\Psi$ be the direct sum of the corresponding inner products. By (2.10) and $(2.11),(F M, \Psi)=(V, \varphi)$. Also by $(2.11), \mathscr{Q}(M)$ contains $L=\mathscr{Q}\left(M_{0}\right), Q=\mathscr{2}\left(\mathbf{M}\left(M_{1}\right)\right)$ and $T=\mathscr{Q}\left(\mathbf{M}\left(M_{2}\right)\right)$, so $\mathscr{Q}(M)=P$.

From (2.11) the ideal class of $M$ is equal to that of $M_{0}$. But $2\left(M_{0}\right)=L$ so the ideal class of $M$ is equal to that of $L$ when $L$ is viewed as a $D$ module.

I wish to thank the referee for suggestions.

\section{REFERENCES}

1. C. W. Curtis and I. Reiner, Representation theory of finite groups and associative algebras, Interscience, New York, 1962.

2. A. Frohlich, On the K-theory of unimodular forms over rings of algebraic integers, Quart. J. Math. Oxford Ser. (2) 22 (1971), 401-423.

3. I. Kaplansky, Linear algebra and geometry, Allyn and Bacon, Boston, Mass., 1969.

4. M. Knebusch, Grothendieck und Wittringe von nichtausgearteten symmetrischen Bilinearformen, S.-B. Heidelberger Akad. Wiss. Math.-Natur. K1. 3 (1969/1970), 93-157.

5. J. Milnor, Symmetric inner products in characteristic 2, Prospects in Mathematics, Ann. of Math. Stud., No. 70, Princeton Univ. Press, Princeton, N.J., 1971, pp. 59-75.

6. J. Milnor and D. Husemoller, Symmetric bilinear forms, Springer-Verlag, Berlin and New York, 1973.

7. O. T. O’Meara, Introduction to quadratic forms, Springer-Verlag, Berlin and New York, 1963.

8. I. Reiner, Maximal orders, Academic Press, London, 1975.

9. A. Roy, Cancellation of quadratic forms over commutative rings, J. Algebra 10 (1968), 286-298.

10. C. H. Sah, A note on Hermitian forms over fields of characteristic 2, Amer. J. Math. 86 (1964), $262-270$.

11. R. C. Wagner, Some Witt cancellation theorems, Amer. J. Math. 94 (1972), 206-220.

12. __ On odd Hermitian forms, J. Algebra 58 (1979), 370-398.

13. G. E. Wall, On the conjugacy classes in the unitary, symplectic and orthogonal groups, J. Austral. Math. Soc. 3 (1963), 1-62.

14. O. Zariski and P. Samuel, Commutative algebra, Van Nostrand, Princeton, N.J., 1958.

Department of Mathematics, Fairleigh Dickinson University, Madison, New Jersey 07940. 